\title{
Abordagem Sistêmica da Gestão de Design com foco no Design de Serviços: uma revisão sistemática.
}

\section{Silva-Borges-da, Diego ${ }^{a}$; Conceição-da-Silva, Rosa Elisa ${ }^{b}$, Berlato, Larissa Fontoura $^{c}$, Silva-Scandolara, Carina ${ }^{d}$, Figueiredo-Gonçalves-de, Luiz Fernando ${ }^{e}$ \& Braviano, Gilson ${ }^{\mathrm{f}}$}

${ }^{a}$ Mestrando em Design, Universidade Federal de Santa Catarina. bordiego@gmail.com

${ }^{\mathrm{b}}$ Doutoranda em Design, Universidade Federal de Santa Catarina. ecsrosa@gmail.com

${ }^{\mathrm{c}}$ Mestranda em Design, Universidade Federal de Santa Catarina. lari.berlato@gmail.com

${ }^{\mathrm{d}}$ Doutoranda em Design, Universidade Federal de Santa Catarina. cariscan@gmail.com

${ }^{\text {e} P r o f e s s o r ~ D o u t o r, ~ P r o g r a m a ~ d e ~ P o ́ s-g r a d u a c ̧ a ̃ o ~ e m ~ D e s i g n .1 f f i g u e i r e d o 2009 @ g m a i l . c o m ~}$

fProfessor Doutor, Programa de Pós-graduação em Design. gilson@cce.ufsc.br

\begin{abstract}
Resumo
Este artigo apresentou uma revisão de literatura, do tipo sistemática, em vistas a mostrar o cenário no campo de pesquisa acadêmica, a partir da relação da Abordagem Sistêmica e da Gestão de Design com foco no Design de Serviço. Assim, o estudo considerou artigos científicos em duas bases de dados: Scopus e Web of Science, resultando em um portfólio bibliográfico de 169 artigos resultantes de uma busca que associou os descritores "systemic approach" AND "design management" AND "service design”. Esses resultados foram agrupados e, posteriormente, filtrados, sendo que ao final do processo dez pesquisas foram revisadas e analisadas. Aspira-se, com este estudo, contribuir para pesquisas futuras na construção de parâmetros que integrem as áreas envolvidas.
\end{abstract}

Palavras chave: Gestão de Design, Design de Serviço, Abordagem Sistêmica, Revisão Sistemática. 


\begin{abstract}
This article presented a literature review, of the systematic type, in order to show the scenario in the field of academic research, starting from the relation of the Systemic Approach and the Design Management with focus on Service Design. Thus, the study considered scientific articles in two databases: Scopus and Web of Science, resulting in a bibliographic portfolio of 169 articles resulting from a search that associated the descriptors "systemic approach" AND "design management" AND "service design". These results were grouped and later filtered, and at the end of the process 10 studies were reviewed and analyzed. The aim of this study is to contribute to future research in the construction of parameters that integrate the areas involved.
\end{abstract}

Keywords: Design Management, Service Design, Systemic approach, Systematic review.

\title{
1. Introdução
}

A economia chamada "pós-industrial" é caracterizada pelo decréscimo da importância da manufatura e aumento da importância da economia de serviços, sendo este, responsável por grande parte da economia mundial, representando 50\% do PIB na Europa, 76\% nos EUA e 40\% na China. No Brasil, segundo o IBGE (2012), em 2011 o setor foi responsável por $69 \%$ do PIB e por $70 \%$ da mão-de-obra empregada no país. Serviços é um tema que demanda ser estudado em maior abrangência e aprofundamento (Lovelock, Wright, 2003).

No contexto de aumento da importância dos fluxos de serviço e informação surge o Design de Serviço, área de estudo dedicada a desenvolver soluções para o setor de serviços. $\mathrm{O}$ Design de Serviço aborda a funcionalidade e a forma de prestação dos serviços a partir da perspectiva do usuário. Seu objetivo é desenvolver as interfaces do serviço de modo que essas sejam úteis, utilizáveis, e desejáveis do ponto de vista do usuário e eficazes, eficientes e distintas do ponto de vista do prestador do serviço (Mager, 2009).

A contribuição da Gestão do Design no Design de Serviços pode se dar através da gestão das relações entre diferentes disciplinas (como design, gestão, marketing e finanças) e diferentes papéis (clientes, designers, equipes de projeto e stakeholders). A função de Gestão do Design é indispensável à criação de qualquer serviço, já que envolve o trabalho 
de diversos profissionais e projetos complexos envolvendo inúmeros pontos de contato entre empresa e pessoas (Best, 2012)

Dessa forma, este estudo sugeriu como hipótese de pesquisa, de que forma a Gestão de Design, por meio de uma Abordagem Sistêmica, pode contribuir com o Design de Serviço? Por meio de uma revisão de literatura, do tipo sistemática, esta pesquisa buscou verificar a produção acadêmica dos eixos de pesquisa de "Abordagem Sistêmica", "Gestão de Design" e "Design de Serviço". Assim, o estudo considerou artigos científicos nas bases Scopus e Web of Science, com objetivo de apresentar o cenário do campo de pesquisa acadêmica dos eixos teóricos supracitados, e, com os objetivos específicos de selecionar um portfólio bibliográfico relevante sobre o tema em questão; realizar uma análise de conteúdo dos artigos deste portfólio e identificar os termos recorrentes alinhados com este estudo.

Por fim, este estudo foi estruturado inicialmente pela fundamentação teórica sobre Gestão de Design, Abordagem Sistêmica e Design de Serviço, necessárias para o direcionamento desta pesquisa; descrição dos procedimentos metodológicos e instrumentos técnicos de pesquisa utilizados; resultados; análise e discussão do portfólio bibliográfico, bem como a descrição sobre cada artigo selecionado e considerações finais.

\section{Fundamentação teórica}

O final do século XX viu surgir uma sociedade e economia baseada no conhecimento, na experiência e nos serviços (Manzini, 2007). Os serviços são as trocas invisíveis que acontecem em todos os lugares e porque eles são um bem intangível e não podem ser possuídos (Shostack, 1984), estão ligadas a experiências, que são pessoais e existem dentro da mente do indivíduo. (Pine; Gilmore, 1998). Para Kotler e Armstrong (1999) "serviço é toda atividade ou benefício, essencialmente intangível, que uma parte pode oferecer a outra e que não tenha como resultado a posse de bens".

O surgimento do Design de serviços é a tentativa de transformar as relações sociais, já que a forma em que a grande maioria dos serviços é planejada e organizada tem gerado grande desgaste do tecido social, destruição e escassez dos recursos ambientais, pobreza e miséria, e pouco tem contribuído para uma verdadeira melhora na qualidade da vida e do bem-estar local. Pois os serviços são sempre pensados como negócios, visando, quase que exclusivamente, o lucro. Então, a ideia do Design de serviços é aproximar e apoiar as empresas e designers a gerenciar a integração entre serviços e negócios, prestando atenção à qualidade da dimensão social e da dimensão sistêmica de serviços (Maffei; Sangiorgi, 2003, Langenbach, 2008). Novas tecnologias utilizadas pela sociedade, voltadas para uma melhoria na qualidade de vida, foram, então, projetadas e organizadas de forma a melhorar 
as relações sociais, projetando o Design de Serviço como uma necessidade de se estabelecer uma visão mais humana de serviços. (Langenbach, 2008).

Nesse contexto, o Design de Serviços carrega um conjunto de áreas de conhecimento, métodos e práticas que permitem que equipes multidisciplinares trabalhem na construção de ofertas de serviços mais adaptadas às pessoas (Pinheiro, 2014). Assim, o designer vê-se como um projetista de melhores soluções na disponibilidade desses serviços, ampliando a satisfação no sentido de que melhora a experiência do cliente.

Bahiana (1998) afirma que, os investimentos voltados para a área de Design deixaram de ser uma questão estética para se tornarem uma questão estratégica. Mozota (2011) corrobora afirmando que o Design é um processo, e não apenas uma forma. A autora sustenta que o Design deve ser relacionado com a beleza de objetos apenas em um segundo nível; em primeiro lugar, tem a ver com toda a abordagem do modo de fazer negócios, servir clientes e oferecer valor.

O Design pode estar presente nos níveis estratégico, tático ou operacional, no estabelecer de objetivos de longo-prazo e em tomadas de decisão do dia-a-dia. O Design é uma função, um recurso, e um modo de pensar, no contexto organizacional, que pode estar presente no pensamento estratégico, no desenvolvimento de processos, e crucialmente, na implementação de projetos, sistemas e serviços. (Best, 2012).

Segundo Cardoso (2013), a contribuição mais significativa que o Design tem a fazer para equacionar os desafios do nosso mundo complexo é o pensamento sistêmico; poucas áreas estão habituadas a considerar os problemas de modo tão integrado e comunicante. $\mathrm{O}$ pensamento sistêmico surge em meio as necessidades de ver forças maiores em jogo dentro da complexidade, também, pela busca em construir descrições coerentes do todo complexo, conforme afirma Andrade et al. (2006).

Silva e Figueiredo (2010) acreditam que a abordagem sistêmica está contribuindo para visualizar um panorama mais abrangente da gestão de Design, entendendo a organização como um sistema aberto, em que as interferências ou problemas detectados podem ser de ordem ambiental, social ou econômica, relativos a interferências e relações internas e externas à organização e ao sistema de Gestão de Design.

A gestão de Design em empresas de serviços pode auxiliar no processo e cultura da organização, na gestão estratégica organizacional, no serviço e interface com o cliente, na comunicação e imagem e na gestão de produtos (Roda e Krucken, 2004). Em um desenvolvimento de serviço é necessário é uma abordagem interdisciplinar capaz de mapear a interação de recursos humanos, tecnologia e organização de forma planejada (Bullinger, 2003). 


\section{Procedimentos metodológicos}

O método de pesquisa sistemática visa realizar uma revisão de literatura que siga um método explícito, planejado, responsável e justificável, conforme Dresch et al. (2015). Nessa perspectiva, os autores afirmam que se trata de "mapear, encontrar, avaliar criticamente, consolidar e agregar resultados de estudos primários relevantes acerca de uma questão ou tópico específicos" e que, ao realizar uma revisão sistemática possibilita-se adquirir uma visão abrangente sobre determinado tema e permite aos pesquisadores colocarem-se a par dos estudos dentro da área de interesse. (Dresch et al., 2015).

Trata-se, portanto, de uma pesquisa exploratória, realizada a partir da busca por dados secundários, ou seja, artigos científicos disponibilizados em bases de dados na internet. Os procedimentos metodológicos compreendem a seleção de fontes de informação para busca de artigos científicos que relacionem as áreas apontadas no problema de pesquisa, que são: Abordagem Sistêmica, Gestão de Design e Design de Serviços. Para os procedimentos da revisão sistemática foram à utilização do Protocolo para Revisão Sistemática da Biblioteca Universitária da Universidade Federal de Santa Catarina (UFSC), disponibilizado no endereço eletrônico da universidade ${ }^{1}$.

A condução de uma seleção de portfólio bibliográfico, a exemplo da desenvolvida nesta pesquisa, tem como objetivo destacar um conjunto de artigos em um processo que ocorre por meio de etapas estruturadas, conforme a seguinte sequência: (1) Definição do problema de pesquisa, (2) Busca na literatura, (3) Avaliação dos resultados e (4) Apresentação dos resultados (relatório).

Na definição do problema, relativo à etapa 1 , foi apresentada a delimitação do tema que será pesquisado balizando assim na condução da pesquisa, bem como os objetivos gerais e específicos que, posteriormente, auxiliaram na análise dos dados coletados com a revisão. A etapa 2, de busca na literatura, foram listados os termos relativos ao tema de pesquisa, e foi nessa fase que foram buscadas as palavras mais adequadas para relacionar termos que congreguem artigos dentro da delimitação do tema de pesquisa descrito na etapa 1 . Foi também na etapa 2 que os critérios de inclusão e exclusão delimitaram a abrangência da pesquisa, como a seleção do tipo de documentos (artigos científicos, livros, dissertações, teses, dentre outros), área geográfica, período de tempo, idioma e quais foram fontes de

\footnotetext{
${ }^{1}$ Durante o desenvolvimento dessa pesquisa foi contatada a Biblioteca Universitária (BU) da UFSC para conhecimento de ferramentas para o desenvolvimento da Revisão Sistemática incluindo capacitações ministradas pelos bibliotecários da BU sobre Bases de Dados e Gerenciadores Bibliográficos. O protocolo utilizado nesta pesquisa está disponível em: $<$ http://portal.bu.ufsc.br $>$.
} 
informações consultadas para a busca. As etapas finais são as de avaliação dos resultados e apresentação dos resultados com a escrita do relatório.

De acordo com Dresh et al. (2015), para as etapas finais do processo existem técnicas de síntese que poderiam ser empregadas em revisões sistemáticas qualitativas, e conforme a classificação apresentada pelos autores, a síntese realizada nesse artigo segue uma linha de síntese textual narrativa, que exibiu um "relato estruturado das características dos estudos, contexto, qualidade e resultados - e da comparação de suas qualidades e diferenças. (Barnett-Page; Thomas, 2009 in Dresch et al., 2015).

\section{Seleção do portfólio bibliográfico}

Tratando-se de três eixos de pesquisa, inicialmente selecionaram-se tópicos para cada eixo da pesquisa, com a aplicação da língua inglesa em todos os tópicos.

a. Primeiro eixo: Systemic Approach e Systemic Thinking.

b. Segundo eixo: Design Management e Strategic Design.

c. Terceiro eixo: Service Design.

A partir de uma busca para teste de aderência dos tópicos, definiu-se pelos tópicos que apresentaram uma quantidade mais representativa de publicações encontradas: Systemic Approach, Design Management e Service Design.

Tabela 1 - Teste de aderência dos tópicos nas bases de dados

\begin{tabular}{|ccc|}
\hline TÓPICOS & $\begin{array}{c}\text { WEB OF } \\
\text { SCIENCE }\end{array}$ & SCOPUS \\
\hline Systemic Approach & 97,164 & 39,337 \\
\hline Systemic Thinking & 17,709 & 1,716 \\
\hline Design Management & 284,813 & 329,872 \\
\hline Strategic Design & 18,121 & 30,717 \\
\hline Service Design & 196,789 & 228,702 \\
\hline
\end{tabular}


As fontes de informação a serem utilizadas nesta pesquisa foram definidas inicialmente pela adequação do conteúdo da base ao tema de pesquisa, a partir de orientação de uma especialista, bibliotecária da Biblioteca Central da UFSC. Em um segundo momento, as fontes de informação foram definidas pela quantidade de artigos disponíveis na área de Design. Assim, optou-se por considerar, para a composição do portfólio bibliográfico, as bases de dados Web of Science e Scopus.

Tabela 2 - Teste das bases de dados na área da pesquisa

\begin{tabular}{|cccc|}
\hline BASES DE DADOS & TÓPICOS & $\begin{array}{c}\text { QUANTIDADE DE } \\
\text { PUBLICAÇOES } \\
\text { ENCONTRADOS }\end{array}$ & $\begin{array}{c}\text { QUANTIDADE DE } \\
\text { ARTIGOS } \\
\text { ENCONTRADOS }\end{array}$ \\
\hline WEB OF SCIENCE & Design & $4,782,250$ & $2,689,447$ \\
\hline SCOPUS & Design & $3,811,377$ & $2,176,574$ \\
\hline
\end{tabular}

$\mathrm{Na}$ primeira busca realizada foram pesquisados os tópicos previamente definidos, Systemic Approach, Design Management e Service Design utilizando-se os operadores booleanos AND entre os termos inseridos no campo topic na Web of Science e no campo article title, abstract, keywords na Scopus, sem nenhuma restrição nos critérios de inclusão/exclusão para o tipo de documento, a área, o período de tempo e o idioma. O resultado encontrado foi de 90 publicações na Web of Science e 122 publicações na Scopus. Na segunda busca, realizada a partir destes resultados e visando refinar a busca para áreas intrínsecas ao Design, utilizou-se a restrição ao domínio de pesquisa para ciências sociais na Web of Science, resultando em 27 publicações e às seguintes áreas de pesquisa na Scopus: negócios, gestão e contabilidade; engenharia; ciências sociais; ciências de decisão; economia; econometria e finanças; artes e humanidades e multidisciplinar, resultando em 60 publicações. Com o objetivo de encontrar publicações mais atuais, refinou-se a busca para os últimos 5 anos (2011-2015), resultando em 15 publicações na Web of Science, destas 13 eram artigos e 28 publicações na Scopus, destas 19 eram artigos. 
Tabela 3 - Resultados das buscas iniciais por tópicos nas bases de dados

\begin{tabular}{|c|c|c|c|c|c|}
\hline BASES DE DADOS & TóPICOS & $\begin{array}{l}\text { QUANTIDADE DE } \\
\text { PUBLICAÇÕES } \\
\text { ENCONTRADAS }\end{array}$ & $\begin{array}{l}\text { COM RESTRIÇĀO } \\
\text { DE ÁREAS- } \\
\text { intrínsecas ao Design }\end{array}$ & $\begin{array}{c}\text { COM } \\
\text { RESTRIC̄ÃO } \\
\text { DE ANO- } \\
\text { 2011-2015 }\end{array}$ & $\begin{array}{c}\text { COM } \\
\text { RESTRIÇĀO } \\
\text { TIPO DE } \\
\text { DOCUMENTO. } \\
\text { artigos }\end{array}$ \\
\hline WEB OF SCIENCE & $\begin{array}{c}\text { (systemic approach) AND } \\
\text { (design management) AND } \\
\text { (service design) }\end{array}$ & 90 & 27 & 15 & 13 \\
\hline SCOPUS & $\begin{array}{c}\text { (systemic approach) AND } \\
\text { (design management) AND } \\
\text { (service design) }\end{array}$ & 122 & 60 & 28 & 19 \\
\hline
\end{tabular}

A quantidade de artigos encontrados, totalizando 32 artigos, foi considerada reduzida. Dessa forma, a etapa seguinte constitui-se de uma breve análise dos títulos dos artigos da primeira e da segunda busca, onde verificou-se a perda de artigos significativos quando aplicado o filtro de restrição de domínio ou área. O Design é multidisciplinar, e tanto a Gestão de Design como o Design de Serviço podem contribuir efetivamente em diferentes áreas. Dessa forma, optou-se por não restringir esta busca sistemática por domínio ou área. Com relação a restrição por período de tempo, a análise dos anos de publicação não se mostrou relevante, visto que a maioria dos artigos foram publicados a partir de 2005.

Reiterando a importância de expandir os resultados desta busca sistemática da literatura, definiram-se sinônimos para cada tópico da pesquisa a partir da leitura das palavras-chave dos artigos encontrados, usando como critério de escolha as palavras mais utilizadas.

a. Primeiro eixo: Systemic Approach. Similares: systemic thinking, systemic view, systemic model, systemic perspective, systemic knowledge.

b. Segundo eixo: Design Management. Similares: design culture, strategic design.

c. Terceiro eixo: Service Design. Similares: service system, service concept, service development, service innovation.

A busca não se mostrou eficiente nas bases de dados Web of Science e Scopus, resultando em erros durante o processamento dos dados devido ao grande número de combinações de palavras. Dessa forma, optou-se por reduzir o número de sinônimos às palavras mais próximas aos objetivos desta pesquisa e manter a utilização destes somente quando a base de dados o permitia.

$\mathrm{Na}$ base de dados Web of Science foram pesquisados os seguintes tópicos: (systemic approach) AND (design management) AND (service design) inseridos no campo topic. $\mathrm{O}$ resultado encontrado foi de 90 publicações e, aplicando-se a restrição para somente artigos, a busca resultou em 71 artigos. Na base de dados Scopus foram pesquisados os tópicos 
(systemic approach) OR (systemic thinking) OR (systemic view) AND (design management) OR (strategic design) AND (service design) OR (service system) OR (service development) no campo article title, abstract, keywords. O resultado foi de 153 publicações e, aplicando-se a restrição para somente artigos, a busca resultou em 98 artigos, totalizando 169 artigos.

Tabela 4 - Resultado da terceira busca por tópicos nas bases de dados

\begin{tabular}{|c|c|c|c|c|}
\hline BASES DE DADOS & TÓPICOS & $\begin{array}{l}\text { QUANTIDADE DE } \\
\text { PUBLICACCÔES } \\
\text { ENCONTRADAS }\end{array}$ & $\begin{array}{c}\text { COM RESTRIÇÃO } \\
\text { TIPO DE } \\
\text { DOCUMENTO - } \\
\text { artigos }\end{array}$ & $\begin{array}{c}\text { TOTAL DE } \\
\text { ARTIGOS } \\
\text { ENCONTRADOS }\end{array}$ \\
\hline WEB OF SCIENCE & $\begin{array}{l}\text { (systemic approach) AND } \\
\text { (design management) AND } \\
\text { (service design) }\end{array}$ & 90 & 71 & \\
\hline SCOPUS & $\begin{array}{c}\text { (systemic approach) OR } \\
\text { (systemic thinking) OR } \\
\text { (systemic view) AND } \\
\text { (design management) OR } \\
\text { (strategic design) AND } \\
\text { (service design) OR (service } \\
\text { system) OR (service } \\
\text { development) }\end{array}$ & 153 & 98 & 169 \\
\hline
\end{tabular}

O primeiro processo de filtragem foi a identificação dos artigos repetidos por meio do uso do gerenciador bibliográfico Mendeley. Nesta etapa foram excluídos por títulos os artigos repetidos, resultando em 148 artigos. A etapa seguinte se refere à leitura dos títulos dos artigos com o objetivo de verificar a adequação destes com o tema da pesquisa, permanecendo 58 artigos que demostraram relevância com o tema de pesquisa.

O terceiro processo de filtragem foi à leitura dos resumos dos 58 artigos selecionados, visando verificar o seu alinhamento com o tema da pesquisa. Em muitos artigos o uso da palavra Design ou Study Design referia-se a abordagem e metodologia utilizada nos artigos; por esse motivo 31 artigos foram descartados foram descartados, totalizando 27 artigos para a leitura completa. Verificou-se, então, a disponibilidade do texto integral destes artigos, onde se identificou três artigos cujos textos não estavam disponíveis gratuitamente, dessa forma não foram avaliados. 
O último processo de filtragem consistiu na leitura integral dos textos dos 24 artigos selecionados, onde 14 artigos foram descartados por não apresentarem alinhamento com o tema de pesquisa. Por fim, o resultado foi de um portfólio bibliográfico de 10 artigos relativos ao tema de pesquisa que são apresentados na Tabela 5.

\section{Tabela 5 - Processos de Filtragem}

\begin{tabular}{|c|c|c|c|c|c|}
\hline $\begin{array}{c}\text { TOTAI. DIS } \\
\text { ARTIGOS } \\
\text { FNCONTRADOS }\end{array}$ & $\begin{array}{l}\text { IXXCLLSÃO } \\
\text { DOS ARTIGOS } \\
\text { REPETIDOS }\end{array}$ & $\begin{array}{c}\text { LEITURA DOS } \\
\text { TITULOS DOS } \\
\text { ARTIGOS } \\
\text { ENCONTRADOS }\end{array}$ & $\begin{array}{c}\text { LEITURA DOS } \\
\text { TÍTUIOS IOS } \\
\text { RESUMOS DOS } \\
\text { ARTIGOS } \\
\text { ENCONTRADOS }\end{array}$ & $\begin{array}{c}\text { DISPONIBILIDADE } \\
\text { DO TEXTO } \\
\text { INTEGRAL DOS } \\
\text { ARTIGOS } \\
\text { ENCONTRADOS }\end{array}$ & $\begin{array}{l}\text { PORTFOLIO } \\
\text { BIBLIOGRÁFICO }\end{array}$ \\
\hline 169 & 148 & 58 & 27 & 24 & 10 \\
\hline
\end{tabular}

Tabela 6 - Portfólio bibliográfico

\begin{tabular}{|c|c|c|c|}
\hline Autores & Título & Ano & Citações \\
\hline $\begin{array}{c}\text { BROZOVIC, D.; RAVALD, } \\
\text { A; NORDIN, F. }\end{array}$ & $\begin{array}{c}\text { Making sense of service dynamics: } \\
\text { the honeybee metaphor. }\end{array}$ & 2015 & 55 \\
\hline SANGIORGI, D. & $\begin{array}{c}\text { Designing for public sector } \\
\text { innovation in the UK: design } \\
\text { strategies for paradigm shifts. }\end{array}$ & 2015 & 62 \\
\hline AKI, J; HARRI, L. & $\begin{array}{c}\text { Distinctive features of service. } \\
\text { performance measurement. }\end{array}$ & 2014 & 51 \\
\hline MORA M; GOMEZ J., & $\begin{array}{c}\text { An Extensive Review of IT Service } \\
\text { Design in Seven International ITSM } \\
\text { Processes Framworks: Part I. }\end{array}$ & 2014 & 7 \\
GELMANG & & \\
\hline EVERARD, M.; McINNES , & $\begin{array}{c}\text { Systemic solutions for multi-benefit } \\
\text { water and environmental } \\
\text { management. }\end{array}$ & 2013 & 36 \\
\hline
\end{tabular}


Silva-Silva, Diego Borges da; Rosa-Rosa, Elisa Conceição da Silva, Berlato- Berlato, Larissa Fontoura, Silva-Silva, Carina Scandolara, Figueiredo- Figueiredo, Dr. Luiz Fernando Gonçalves de \& Braviano- Braviano, Dr. Gilson.

\begin{tabular}{|c|c|c|c|}
\hline ZAREI S; MALAYERI A. D. & $\begin{array}{c}\text { Data Management System and } \\
\text { Knowledge Analyzing via KADS } \\
\text { Approach. }\end{array}$ & 2012 & 3 \\
\hline CEPPA C. & $\begin{array}{c}\text { Systemic Software: an IT network at } \\
\text { the service of the environment. }\end{array}$ & 2010 & 3 \\
\hline $\begin{array}{c}\text { HINRICHS, S; DICKERSON, } \\
\text { T; CLARKSON, P.J. }\end{array}$ & $\begin{array}{c}\text { A Case study of Design Methods } \\
\text { Applied to Researching Medical } \\
\text { Device Purchasing Systems. }\end{array}$ & 2010 & 2 \\
\hline SHIER, M; WALSH, C. A; & $\begin{array}{c}\text { Conceptualizing Optimum Homeless } \\
\text { Shelter Service Delivery: The } \\
\text { Interconnection between } \\
\text { Programming, Community, and the } \\
\text { Built Environment. }\end{array}$ & 2007 & 14 \\
\hline STEVIMITIS. R. & $\begin{array}{c}\text { Managing the new service } \\
\text { development process: towards a } \\
\text { systemic model. }\end{array}$ & 2005 & 61 \\
\hline
\end{tabular}

\section{Seleção do portfólio bibliográfico Análise e revisão dos resultados}

O processo de análise e revisão dos resultados está organizado conforme a sequência da tabela 6, apresentada anteriormente, que segue a ordenação dos artigos por ano, de publicações mais recentes para as mais antigas. $\mathrm{O}$ relato dos dados foi estruturado com a apresentação sucinta do artigo e contextualização do assunto com suas aproximações aos eixos teóricos da temática dessa pesquisa.

A pesquisa de Brozovic, Ravald e Nordin (2015) sobre Design de Serviço diz respeito a metáfora da colônia de abelhas como ferramenta analítica e discursiva para compreender os aspectos dinâmicos de sistemas de serviços em torno de uma relação de serviço. De modo sistêmico, os profissionais de marketing personificam "abelhas", assistem as "flores", ou seja, os clientes, fornecendo "polinização", assegurando o fluxo de processos dos clientes, atendendo a sua oferta, que é uma parte integrante dos processos. Mesmo uma atividade de polinização - tarefa das mais simples - pode instigar a transferência de informações e transformá-la em uma nova oferta de serviços. Essa organização é semelhante à forma 
como uma colônia de abelhas adapta a informação recebida de abelhas individuais e, subsequentemente, adapta suas ações.

O estudo de Sangiorgio (2015) envolveu o governo e a população do Reino Unido com o propósito de refletir uma mudança do modelo New Public Management para o modelo New Public Governance, visando abordagens alternativas de inovação na prestação de serviços. Esse novo modelo de gestão implicou em uma ascensão de redes e parcerias, bem como uma co-produção como modelo de serviços, apresentando vantagens evolutivas na aprendizagem e inovação, visto que parcerias e redes entre os setores públicos, privados e voluntários eram considerados o locus da inovação.

Compreender as particularidades da medição de desempenho de serviço foi o foco do trabalho de Aki e Harri (2014). Por meio de uma análise da visão geral de três setores de serviços na Finlândia - industriais; conhecimento intensivo; público - os autores buscaram compreender as características de medição de desempenho distintas nos setores de serviços escolhidos. Nesse sentido, Gestão e Serviço são dois conceitos abordados como componentes-chave para a medição de desempenho orientada para o cliente, destacando o papel do consumidor, não só como um avaliador do desempenho dos serviços, mas também como participante no desenvolvimento de instrumentos de medição que sejam válidos.

A pesquisa de Mora et al. (2014) teve como objetivo o avanço na compreensão sobre o estado da arte da Gestão de Serviço de TI (ITSM - IT Service Management) e de como este pode ser projetado. Diversos são os impactos positivos da ITSM, entre eles melhoria: na qualidade, produtividade e eficiência dos serviços de TI; na visibilidade dos processos de TI; na consistência e documentação do processo de Gestão de Serviço; no gerenciamento de incidentes, mudanças e práticas de testes; no aumento da satisfação do cliente e na resposta e tempo de resolução e identificação clara dos papéis e responsabilidades. Concluiu-se que os principais processos internacionais de Gestão de Serviços de TI incluem o Design de Serviço de TI como parte de suas melhores práticas.

Os autores Everard e Mcinnes (2013) apresentaram a necessidade de otimização em serviços ecossistêmicos, entendendo que o conceito de "soluções sistêmicas" pode ajudar a abordagem ao ecossistema sob a forma de soluções sustentáveis para problemas de gestão ambiental. Para os autores, "soluções sistêmicas" seriam tecnologias de entrada utilizando processos naturais para otimizar serviços de água, evitando impactos negativos e uma ampla mudança de cultura para uma prática mais sustentável.

O Sistema de Gestão de Distribuição (DMS) é amplamente desenvolvido para sistemas de planejamento, design, instalação, comissionamento, operação e período de manutenção, visando controlar e monitorar a rede de distribuição para aumentar o desempenho e reduzir custos operacionais e de manutenção. O estudo de Zarei e Malayeri (2012) propôs uma abordagem do Pensamento Sistêmico e de metodologias da Engenharia do Conhecimento 
Silva-Silva, Diego Borges da; Rosa-Rosa, Elisa Conceição da Silva, Berlato- Berlato, Larissa Fontoura, Silva-Silva, Carina Scandolara, Figueiredo- Figueiredo, Dr. Luiz Fernando Gonçalves de

utilizadas como ferramenta colaborativa no Design de DMS. O estudo do Design do DMS da Autoridade Metropolitana de Eletricidade (MEA - Metropolitan Electricity Authority) foi utilizado para demonstrar a aplicabilidade e a vantagem do uso do pensamento sistêmico e do Common KADS para a obtenção de dados e informações de uma forma sistemática.

A pesquisa de Hinrichs, Dickerson e Clarkson (2010) apresentou um exemplo da aplicação de métodos e ferramentas de Design sistêmico na prática de compra de dispositivos médicos no Serviço Nacional de Saúde do Reino Unido (NHS). O estudo explorou as abordagens de Design como parte do processo de investigação, em termos de recolha de dados, análise, síntese, bem como na implementação de novas práticas. A abordagem sistêmica mostrou-se de grande valia em um contexto onde vários stakeholders estão envolvidos no processo de decisão visando atingir os mesmos objetivos, mas com diferentes formações e objetivos em suas linhas de trabalho.

Ceppa (2010) apresentou um instrumento de TI com um conceito de circuito aberto para auxiliar as empresas em seus processos de produção e descarte de resíduos. Esse instrumento possibilitaria uma transformação concreta de resíduos em materiais de modo digno, rentável e reutilizável. O Software Sistêmico para gerar novas "redes ecológicas" é um instrumento que pode gerar análises de sistemas com abordagem sistêmica podendo apoiar companhias em trocas de recursos e competências com consequente ganho para todos os operadores envolvidos na rede de relacionamento.

O estudo de Shier, Walsh e Graham (2007) teve como objetivo principal a obtenção de informações sobre a prestação de serviços de abrigos para moradores de rua nos EUA e no Reino Unido; buscando ampliar a investigação social para além do trabalho sobre o ambiente social, atendendo também o ambiente físico, contribuindo assim no desenvolvimento de futuras inovações. Os resultados identificaram as principais características necessárias para sucesso na prestação deste tipo de serviço: características sistêmicas do abrigo, como programas do abrigo que promovam a educação e o treinamento profissional; a gestão do abrigo, dignidade e segurança do cliente; relacionamento com a comunidade, como segurança pública, mudança de percepção da comunidade sobre os moradores de rua e envolvimento positivo de toda a comunidade; e o ambiente construído, que diz respeito à arquitetura do abrigo, integração dessa ao ambiente urbano local, o tamanho, a localização, a acessibilidade e o impacto de um abrigo na comunidade. Cada um desses fatores se encontra interligado de uma forma complexa, aspectos do ambiente construído impactam no sistema de serviços do abrigo que, por sua vez, pode ter implicações negativas para as relações comunitárias. Conclui-se a necessidade de uma abordagem sistêmica no desenvolvimento e prestação desse tipo de serviço. 
Stevens e Dimitriadis (2005) apresentaram um estudo de caso sobre um novo serviço de gestão para o desenvolvimento de diferentes ofertas para bancos e varejo. $\mathrm{O}$ estudo revelou mudanças nos cenários de entrega intangíveis apoiados pelo redesign do sistema de informação, bem como as infraestruturas; um processo de tomada de decisão alcançada por meio de um processo de aprendizagem organizacional que transforma as intuições individuais e conflitos cognitivos em rotinas organizacionais por interações sucessivas; e um processo de mudança que dura até a implementação dos procedimentos na distribuição rede, para adaptá-los às suas próprias experiências. Eles apontam que o caráter sistêmico se apresenta também nas interações tanto entre humanos quanto aos dispositivos técnicos que contribuem com o processo de interação.

Dos artigos selecionados e analisados no portfólio bibliográfico desta revisão, percebeu-se convergências durante as leituras e análises, principalmente em aspectos como os da relação entre a Abordagem Sistêmica e processos de aprendizagem; sobre necessidade de troca e compartilhamento de informações; no uso de ferramentas para lidar com as complexidades relativas a gestão, item que salientou a necessidade da visão sistêmica para a solução de problemas; a criação de redes entre empresas, parceiros e colaboradores, reforçando a atenção sobre os stakeholders, indicando práticas de co-produção para modelos e serviços, design sistêmico e design participativo na relação com os consumidores/clientes e demais envolvidos nos processos.

\section{Considerações finais}

Um dos principais desafios para o Design de serviços, enquanto área de pesquisa, é definir sua estrutura: encontrar e desenvolver teorias e métodos que tornem a pesquisa criticável. O Design de serviços precisa encontrar formas de estruturar e apresentar conhecimento, de modo a permitir que designers utilizem esse conhecimento e que os pesquisadores continuem a desenvolver conhecimento cumulativamente (Stickdorn e Schneider 2010).

O propósito desta revisão sistemática foi identificar, a partir de uma criteriosa seleção, pesquisas científicas que pudessem responder ao tema inicial da pesquisa, que era investigar como a Gestão de Design, por meio de uma Abordagem Sistêmica, pode contribuir com o Design de Serviço.

Stickdorn e Schneider (2010) afirmam que o Design e a gestão, quando pensados em conjunto, podem gerar resultados construtivos na oferta de serviços inovadores. Os autores acreditam que nos esforços de fundamentar a pesquisa na área de Design de serviço a partir dos conhecimentos de áreas relacionadas, destaca-se o campo da gestão.

Diante da quantidade de estudos recuperados, 169 ao todo, foi possível perceber que as temáticas abordadas estão presentes em diversos estudos e em diversas áreas, sendo que os 
Silva-Silva, Diego Borges da; Rosa-Rosa, Elisa Conceição da Silva, Berlato- Berlato, Larissa Fontoura, Silva-Silva, Carina Scandolara, Figueiredo- Figueiredo, Dr. Luiz Fernando Gonçalves de

dados e as informações inferidas foram comprovados, por critérios de exclusão e refinamento nas bases Scopus e Web of Science, totalizando, ao final do processo, dez artigos que foram selecionados para leitura e, posteriormente, analisados e que geraram insights descritos na parte cinco deste estudo.

Sugere-se, numa próxima etapa de pesquisa, identificar estudos desenvolvidos aqui no Brasil, visto que durante as etapas de seleção e filtragem dos artigos, nenhum autor brasileiro foi identificado. Também, recomendações para estudos sobre indicativos bibliométricos.

\section{Referências}

Andrade, A. L. et al. (2006). Pensamento istêmico: o desafio da mudança sustentada nas organizações e na sociedade. Porto Alegre: Bookman.

Aki, J; Harri, L. (2014). Distinctive features of service performance measurement. International Journal of Operations \& Production Management. v. 34. Iss 12. p. 1466 - 1486. Recuperado de http://www.emeraldinsight.com/doi/abs/10.1108/IJOPM-02-2013-0067

Bahiana, C. (1998). A import $n$ cia do e sign para sua empresa. CN, CO P, NA R -RJ, Brasília, DF. CNI.

Best, K. (2012). Fundamentos da Gestão do Design. Tradução: André de Godoy Vieira. Porto Alegre: Bookman.

Brozovic, D; Ravald, A; Nordin, F. (2015). Making sense of service dynamics: the honeybee metaphor. Journal of Services Marketing. v. 29. ISS 6/7. p. 634 - 644. Recuperado de http://www.emeraldinsight.com/doi/abs/10.1108/JSM-01-2015-0046

Bullinger, H. (2003). Service engineering - methodical development of new service products. International Journal of Production Economics, v. 85, n. 3, p. 275-287.

Cardoso, R. (2013). Design para um mundo complexo. São Paulo: Cosac Naify. CEPPA C. (2010) Systemic Software: an IT network at the service of the environment. In: DESIGN PRINCIPLES AND PRACTICES, v. 4, pp. 345-354.

Hinrichs, S; Dickerson, T; Clarkson, P. J. (2010). A Case study of Design Methods Applied to Researching Medical Device Purchasing Systems. Australasian Medical Journal, v. 3, n. 8, p. 471-487. Recuperado de http://www.amj.net.au/index.php?journal=AMJ\&page=article\&op=viewFile\&path $\% 5 \mathrm{~B} \% 5$ $\mathrm{D}=401 \&$ path $\% 5 \mathrm{~B} \% 5 \mathrm{D}=633$ 
Abordagem Sistêmica da Gestão de Design com foco no Design de Serviços: uma revisão sistemática.

Kotler, P; Armstrong, G. (1999). Princípios de Marketing. Tradução: Vera Whately, revisão técnica: Roberto Meireles Pinheiro. 7.ed. Rio de janeiro: LTC.

Langenbach, M. L. (2008). Além do apenas funcional: inovação social e design de serviços na realidade brasileira. 2008. 123p. Dissertação de Mestrado em Ciências em Engenharia de Produção. Departamento de Engenharia. Universidade Federal do Rio de Janeiro - UFRJ, Rio de Janeiro, Brasil.

Lovelock, C; Wright, L. (2003). Serviços: Marketing e Gestão. São Paulo: Saraiva.

Mager, B. (2009). Service Design as an Emerging Field. In: S. Miettinen; M. Kivisto; Designing Services with Innovative Methods. Helsink: Helsinki Univesity of Art and Design.

Manzini, E. (2007) Sustainability: systemic change and social learning process.

Mora M; Gomez J., O’Connor R; Raisinghani M; Gelman O. (2014). An Extensive Review of IT Service Design in Seven International ITSM Processes Framworks: Part I. International Journal of Information Technologies and Systems Approach. v. 7. n. 2, p. 83 - 107. Recuperado de http://doras.dcu.ie/20308/1/oconnor_article_JITSA_7(2).pdf

Mozota, B. B. de. (2011). Gestão de Design: usando o Design para construir valor de marca e inovação corporativa. Porto Alegre: Bookman.

Pine, B.; Gilmore, J. (1998). Welcome to the experience economy. Harvard Business Review, Julhoagosto, pp. 97-105.

Pinheiro, T. (2014). The Service Startup: Design gets lean. United States: Amazon.com.

Roda, R; Krucken, L. (2004). Gestão do Design aplicada ao modelo atual das organizações: Agregando valor a serviços. In: Proceedings P\&D 2004 - 6o Congresso Brasileiro de Pesquisa e Desenvolvimento em Design, 2004, Fundação Armando Alvares Penteado FAAP, São Paulo.

Sangiorgi, D. (2015). Designing for public sector innovation in the UK: design strategies for paradigm shifts. Foresight. v. 17. ISS 4. p. 332 - 348. Recuperado de http://dx.doi.org/10.1108/FS-08-2013-0041

Shier, M; Walsh, C. A; Graham, J. R. (2007). Conceptualizing Optimum Homeless Shelter Service Delivery: The Interconnection between Programming, Community, and the Built Environment. Canadian Journal of Urban Research. v. 16, n. 1; p. 58 - 75. Recuperado de https://www.researchgate.net/publication/224937414_Conceptualizing_optimum_homeless _shelter_service_delivery_The_interconnection_between_programming_community_and_t he_built_environment

Shostack, G. L. (1984). Designing services that deliver. Harvard Business Review. USA, v. 62, n. 1, p. 133-139.

Silva, C. S. da; Figueiredo, L. F. G. de. (2010). Abordagem Sistêmica da Gestão de Design em Microempresas e Empresas de Pequeno Porte (MPEs). In: 9o Congresso Brasileiro de 
Silva-Silva, Diego Borges da; Rosa-Rosa, Elisa Conceição da Silva, Berlato- Berlato, Larissa Fontoura, Silva-Silva, Carina Scandolara, Figueiredo- Figueiredo, Dr. Luiz Fernando Gonçalves de \& Braviano- Braviano, Dr. Gilson.

Pesquisa e Desenvolvimento em Design, São Paulo, p.730-741. Recuperado de $<$ http://blogs.anhembi.br/congressodesign/anais/artigos/69903.pdf $>$

Stickdorn, M.; Schneider, J. (2010). This is Service Design Thinking. BiS Publishers. Amsterdam.

Zarei S; Malayeri A. D. (2012). Data Management System and Knowledge Analyzing via KADS Approach. Middle-East Journal of Scientific Research. v. 11, n. 5, p. 595-601. Recuperado de http://www.idosi.org/mejsr/mejsr11(5)12/9.pdf 